\title{
Outcome of a Ceratohyiodectomy in a Criollo Mare with Temporohyoid Osteoarthropathy
}

\author{
Mariana Andrade Mousquer', Leandro Américo Rafael ${ }^{1}$, Nathalia de Oliveira Ferreira', \\ Margarida Aires da Silva', Taís Scheffer del Pino', Cassiano Portela de Assis², \\ Bruna da Rosa Curcio' \& Carlos Eduardo Wayne Nogueira'
}

\begin{abstract}
Background: Temporohyoid osteoarthropathy (THO) is a progressive disease that causes acute onset of peripheral vestibular signs with or without facial paralysis. Ankylosis of temporhyoid joint occurs which predispose to fractures of the involved bones and consequently causes the commonly neurological signs observed. Clinical signs vary depending on the stage of the disease and the nerves affected. Surgical treatment is advised to improve survival rates in which the ceratohyoidectomy is currently known as the most advantageous. The aim of the present study is to report a case and outcome of a ceratohyoidectomy procedure in a Criollo mare presenting THO of the right temporohyoid joint.

Case: A 17-year-old Criollo mare was referred to the Equine Clinical Hospital of the Federal University of Pelotas with a 5-day history of facial paralysis on the right side, head tilt and difficulty to chew and swallow. Auricular, palpebral and labial ptosis along with deviation of the lip and nostril to the left were observed. A corneal ulcer was also identified in the right eye. Complementary imaging exams (endoscopy of the guttural pouches and radiography of the head) were performed and showed thickening of the right stylohyoid bone confirming a diagnosis of THO. Anti-inflammatory and antibiotic therapy were administered and the corneal ulcer was treated with topical antibiotics and autologous serum. Due to rapid deterioration of clinical signs, the mare was referred to surgery. A ceratohyoidectomty procedure was performed under general anesthesia. In this procedure, the ceratohyoid bone was disarticulated from the ceratohyoid-basihyoid joint and removed. During the procedure, a branch of the linguofacial vein was accidentally incised causing hemorrhage, the branch was identified and successfully ligated. Recovery was uneventful. Supportive treatment with anti-inflammatory and antibiotics was continued after surgery and two sessions of electro-acupuncture was also performed to improve the nerve paralysis. The electro-acupuncture was discontinued due to mare's negative behavior on needle insertion in the face. The treatment of the ulcer was changed since no improvement was observed in the first days. Twenty-eight days after hospitalization, the mare was discharged with the ulcer healed and significant improvement of neurological signs. A complete recovery occurred within three months.

Discussion: The Criollo mare was referred to the hospital presenting mild neurological signs consistent with vestibular alteration and facial nerve paralysis. The THO diagnosis was confirmed using complementary imaging exams in which the endoscopy of the guttural pouch is considered the most common when computed tomography, a more sensitive one, is not available. Unilateral ceratohyoidectomy was performed as a surgical choice of treatment since it has a higher survival rate and lower recurrence rate in comparison to medical treatment and to stylohyoidectomy. As the main intraoperative complication, a vessel was accidentally incised, however this is described to occur in some cases. Despite that, the procedure was successfully performed and the mare had a complete recovery of the neurological signs and corneal ulcer. In conclusion, this report showed that it is important to have a complete diagnosis of these diseases and a consistent treatment plan to improve patient's survival and quality of life.
\end{abstract}

Keywords: neurologic disease, peripheral vestibular signs, facial paralysis, ceratohyoid bone, ceratohyoidectomy. 
M.A. Mousquer, L.A. Rafael, N.O. Ferreira, et al. 2021. Outcome of a Ceratohyiodectomy in a Criollo Mare with Temporohyoid

\section{INTRODUCTION}

Temporohyoid osteoarthropathy (THO) affects the temporohyoid joint (THJ) and is the most common cause of acute onset of peripheral vestibular signs and/ or facial nerve paralysis $[1,8]$. Despite some hypotheses, the etiopathology, the etiopathology of this disease is still unknown $[1,4,19]$. THO causes degenerative changes in the THJ due to chronic bony proliferation of the stylohyoid bone and the petrous temporal bone, leading to ankylosis of the joint [18]. After fusion, the flexibility of the entire hyoid apparatus decreases and abnormal stress is put in the involved structures, which can cause fracture and the acute neurological symptoms mostly observed $[8,14]$.

Clinical signs can vary depending on the stage of the disease and the subsequent nerves affected. It can be identified since behavior problems, difficult to chew up until signs of vestibular disease like head tilt, nystagmus and severe loss of balance [4]. Diagnosis relies on clinical signs and complementary imaging exams $[10,11,21]$. Treatment should address inflammation, any secondary problems due to nerve dysfunction and release the pressure of THJ to decrease the pain [4]. Although a more conservative medical treatment is described, surgical approach is advised to improve survival chances [5]. The ceratohyoidectomy (CHO) is the preferred surgical procedure currently chosen [5].

There are several reports of ceratohyoidectomy for treatment of $\mathrm{THO}$ in different countries
$[2,5,13-16,19,21]$, however, to the author's knowledge, this is the first report of this procedure in Brazil. Therefore, the aim of this study is to report a case and outcome of a ceratohyoidectomy procedure in a Criollo mare presenting THO of the right THJ.

\section{CASE}

A 17-year-old Criollo mare was referred to the Equine Clinical Hospital of the Federal University of Pelotas (HCV- UFPel) with a 5-day history of paralysis on the right side of the face with a head tilt to the same side and subsequent difficulty to chew and swallow. At the initial general clinical exam all the vital parameters were within normal reference range for the species [20]. In a more specific clinical evaluation of the head, auricular, palpebral and labial ptosis along with deviation of the lip and nostril to the left were identified indicating unilateral paralysis of the facial nerve on the affected side. Epiphora was also present in the right eye, which was stained with fluorescein dye and a superficial corneal ulcer was observed.

Radiographs of the head were performed and thickening of the right stylohyoid bone was identified in the dorso-ventral and latero-lateral position (Figure 1). Endoscopy of the upper respiratory tract was also performed where narrowing of the nasopharyngeal ostium of the right guttural pouch was observed when compared to the contralateral side and thickening stylohyoid bone (Figure 2) was confirmed once the inside of the guttural pouch was accessed, affirming a diagnosis of THO.

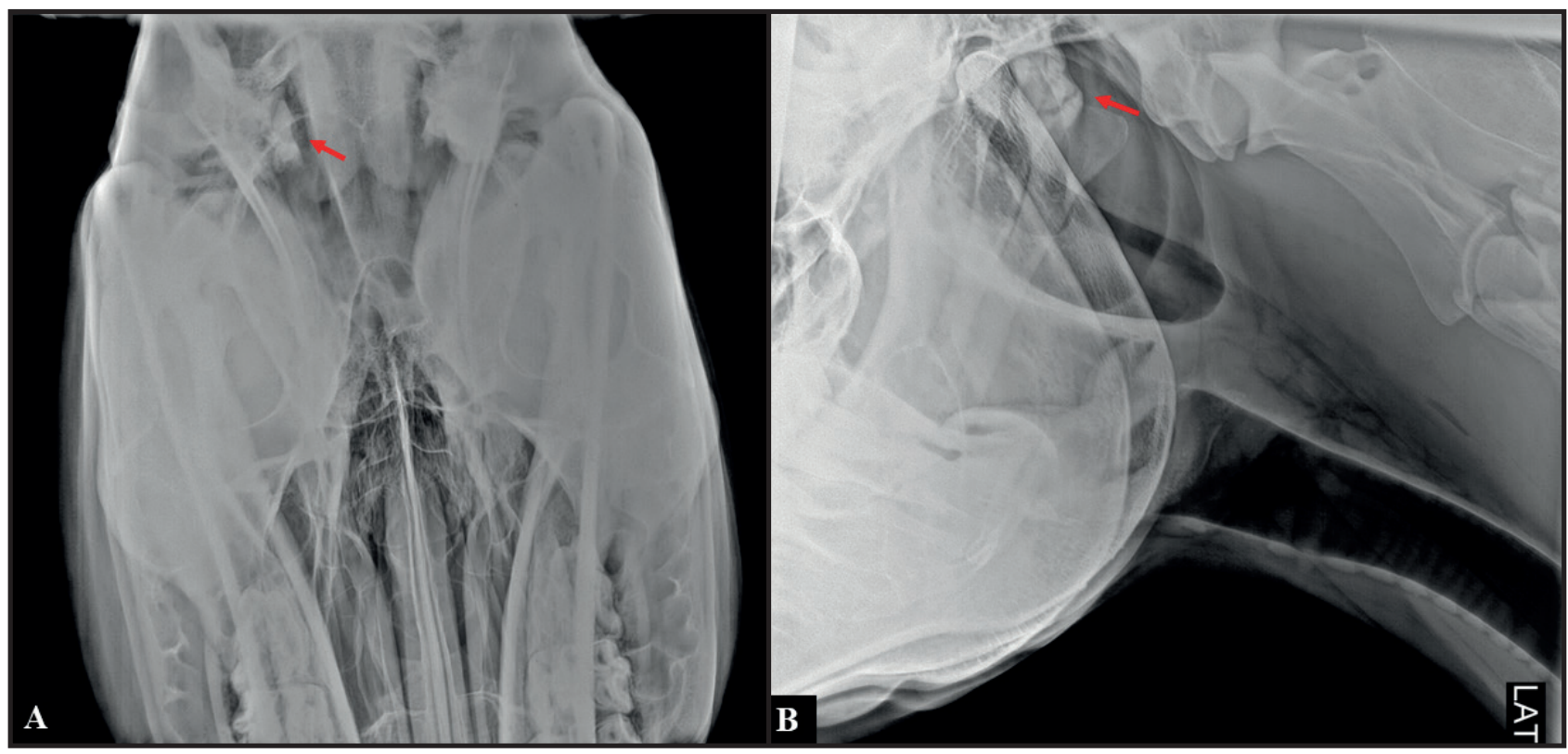

Figure 1. A- Dorso-ventral radiograph of the head showing the affected temporohyoid joint (red arrow). B- Lateral radiograph of the head showing the affected joint (red arrow). 


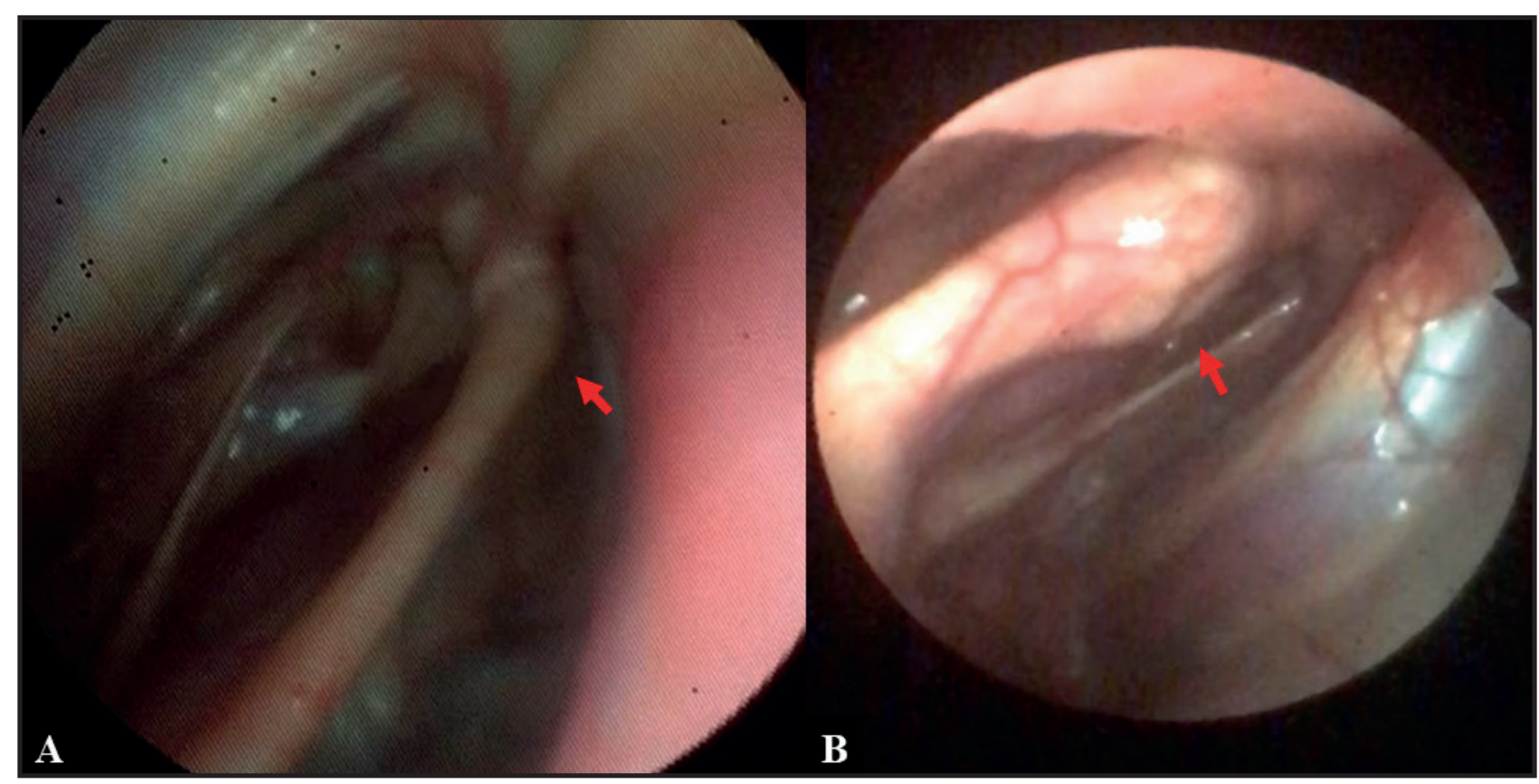

Figure 2. Comparative endoscopy of both guttural pouches. A- Left guttural pouch showing normal stylohyoid bone and THJ (red arrow). B- Right guttural pouch showing thickening of the stylohyoid bone (red arrow).

Initial therapy consisted of Meloxicam [Maxican $\left.^{1} 2 \%, 0.6 \mathrm{mg} / \mathrm{kg}, \mathrm{IV}\right]$ and Dimethylsulfoxide [Dimesol ${ }^{2}, 10 \%$, IV for 5 days]. For the corneal ulcer topic treatment with tobramycin [Tombramicina ${ }^{3}$ every $2 \mathrm{~h}$ ] and autologous serum [every $4 \mathrm{~h}$ ] was established. A subpalpebral lavage system had to be inserted for continuous treatment of the eye. The mare was maintained in medical treatment for 5 days due to the owner's decision, however, it started presenting mild incoordination and nystagmus of the right eye. At that time, due to the worsening of the neurologic signs, the owner consented to surgery and the mare was referred for a unilateral ceratohyoidectomy procedure.

As preoperative medication, flunixin meglumine [Flumedin ${ }^{4}, 1.1 \mathrm{mg} / \mathrm{kg}$ ], gentamycin [Gentatec ${ }^{5}$, $6.6 \mathrm{mg} / \mathrm{kg} \mathrm{IV}$ ] and benzathine penicillin [Penfort PPU ${ }^{1}$, $20.000 \mathrm{UI} / \mathrm{kg} \mathrm{IM}]$ were given. The mare was sedated with xylazine [Equisedan ${ }^{6}, 0.8 \mathrm{mg} / \mathrm{kg}$ ] and anesthesia induction was performed with ketamine [Cetamin 7 , $2.2 \mathrm{mg} / \mathrm{kg}$ ] and diazepam [Diazepam ${ }^{8}, 0.1 \mathrm{mg} / \mathrm{kg}$ ]. Isoflurane (Isoforine ${ }^{9}$ ) was used for maintenance. The mare was placed in dorsal recumbency and the ventral area of the larynx and intermandibular space was clipped and aseptically prepared with chlorhexidine $2 \%$ $(\text { Clorexidina } 2 \%)^{10}$. An incision of approximately $10 \mathrm{~cm}$ long was made $2 \mathrm{~cm}$ apart from the ventral midline just above the basihyoid bone in the right side. The sternohyoid muscle was exposed and separated until the lingual process and then the basihyoid bone were identified. The geniohyoid muscle was also separated to exhibit the ceratohyoid bone. The hypoglossal nerve was identified and gently retracted to access the ceratohyoid-basihyoid joint. When this joint was being disarticulated, a branch of the linguofacial vein was accidentally incised causing extensive bleeding, however, the branch was quickly identified and hemostasis was successfully realized. The removal of ceratohyoid bone proceeded and the remaining muscular portion and skin were sutured. Recovery from anesthesia was uneventful and no immediate postoperative complications were observed.

Postoperative medication consisted of Meloxicam [Maxican 2\% $\%^{1}, 0.6 \mathrm{mg} / \mathrm{kg}, \mathrm{IV}$, Sid for more 5 days], benzathine penicillin [Penfort PPU ${ }^{1}, 20.000 \mathrm{UI} / \mathrm{kg}$, IM) every $48 \mathrm{~h}$ for 3 times, gentamycin [Gentatec ${ }^{5}$, $6.6 \mathrm{mg} / \mathrm{kg}$, Sid for 5 days], sucralfate [Sucralfato ${ }^{11}, 4 \mathrm{~g}$, PO, bid for 3 days]. The subpalpebral lavage system was withdrawn and the ophthalmic treatment was changed for topic moxifloxacin [Vigamox ${ }^{12}$ every $4 \mathrm{~h}$ ], an ophthalmic ointment was started [Regencel ${ }^{9}$ every $4 \mathrm{~h}$ ] and autologous serum was continued for more 7 days.

Electro acupuncture was also performed aiming to stimulate facial nerves, however, due to mare's negative behavior to insert the needles, only two sessions were completed. After 28 days of treatment, the mare was discharged from the hospital with significant improvement of all neurological signs including improvement 


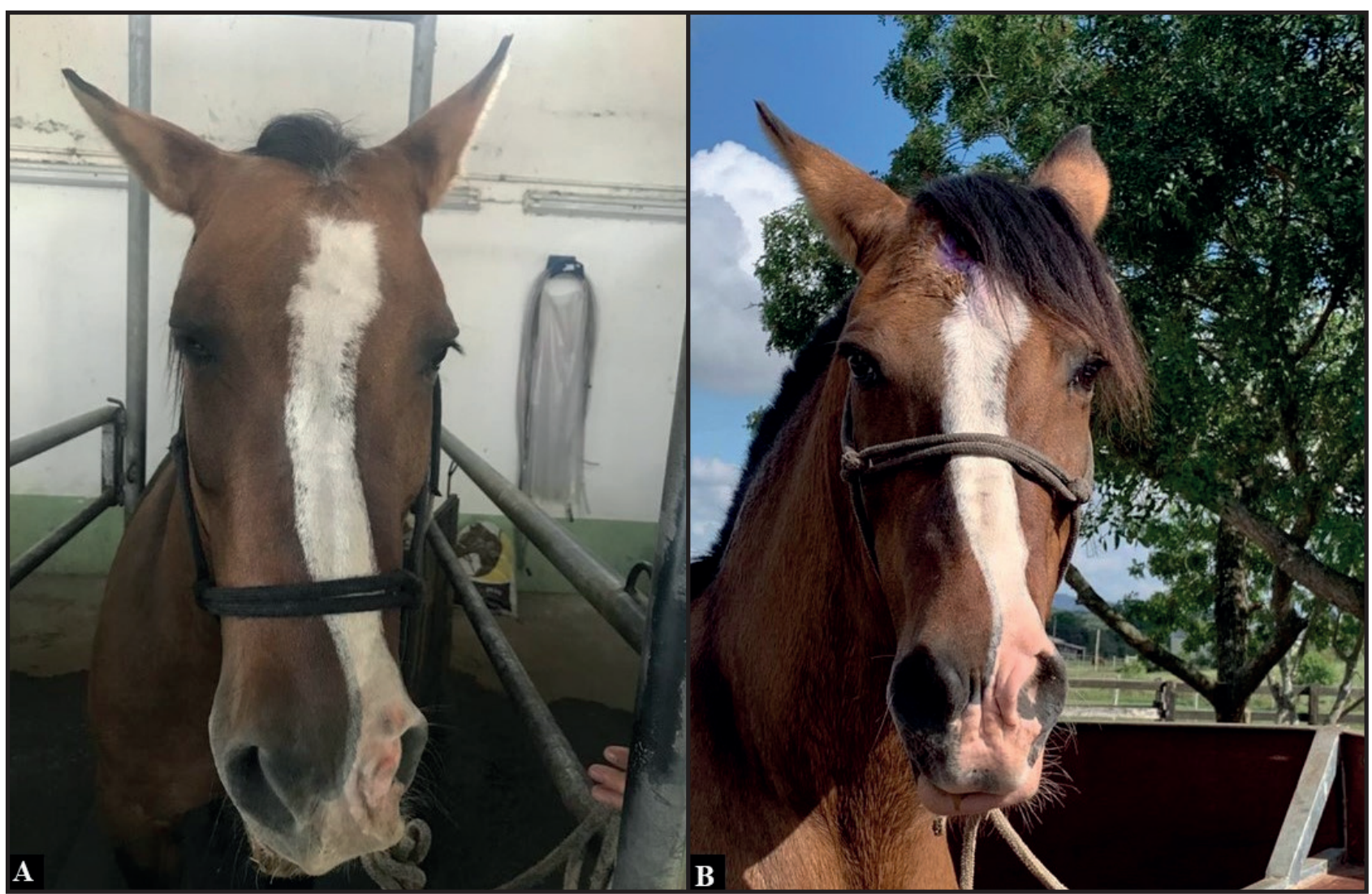

Figure 3. A- Mare presenting signs of right facial paralysis with auricular, palpebral, labial ptosis and deviation of the lip and nostril towards left. B- Day of discharge, the mare shows improvement of facial paralysis signs.

to chew and swallow, incoordination, nystagmus and facial paralysis (only a mild ptosis of the ear was still present) and the ulcer was completely healed (Figure 3). A follow up examination was performed 3 months from the discharge by the referring veterinarian, which observed complete recovery of all clinical signs.

\section{DISCUSSION}

The mare presented in this report had mild neurological symptoms consistent with peripheral vestibular alteration and facial nerve paralysis which led to complementary investigation to confirm a diagnosis of THO. Endoscopic evaluation of the guttural pouch is considered the most commonly diagnostic tool utilized to confirm THO by which is possible to evaluate symmetry of both stylohyoid bones and the THJ [18,21]. Radiographs from the head can also be performed to evaluate stylohyoid bones and THJ, however, when no abnormalities are found in this exam, it does not rule out the presence of the disease [21]. In the Criollo mare reported here, radiography and endoscopy exams were performed and thickening of the right stylohyoid bone was identified in both.
Computed tomography (CT) has also been described as more advantageous for THO diagnosis $[2,10,11]$, however, the exam was not available to be performed in the present patient. In a study by Hilton et al. [10], CT identified abnormalities that were not present in the radiographic and endoscopic exams of patients with THO. The authors also observed in that study that even though all the horses evaluated had unilateral clinical signs, bilateral alterations were recognized in the CT [10].

The Criollo mare had unilateral clinical signs, endoscopic and radiographic alterations. It is known that neurological signs are usually unilateral even when both joints are affected [10,12]. Bras, Davis $\&$ Beard [2] reported a case of a horse affected with THO bilaterally in which they had to perform the ceratohyoidectomy procedure in both sides with an interval of two years apart. However, another study demonstrated that a greater number of horses affected bilaterally underwent unilateral $\mathrm{CHO}$ procedure, performed in the most affected side, improving clinical signs significantly and not requiring another surgical intervention [5]. 
Unilateral ceratohyoidectomy was the surgical choice of treatment for the mare of this report as this procedure is currently the most advantageous described in literature $[5,13]$ and is also reported to have a higher survival rate than cases treated only medically [5]. Partial stylohyoidectomy was also described as a surgical option for THO [1], however, it is known to cause more complications and to have a higher recurrence rate of clinical signs due to regrowth of the stylohyoid bone [15]. The ceratohyoidectomy aims to reduce the forces on the THJ subsequently reducing the pain and clinical signs associated with bone fracture and proliferation [16,21].

The surgical procedure was performed as described elsewhere [6], however, as a complication, a vessel was accidentally incised. Although profuse hemorrhage in the surgical field occurred, the vessel was clamped and ligated. This was the main intraoperative complication in our patient which was also reported to occur in other cases $[13,16]$. Other possible complications for $\mathrm{CHO}$ include damage of neural structures and some horses can experience respiratory distress shortly after anesthesia recovery requiring temporary tracheostomy [5,16]. Racine et al. [16] described the $\mathrm{CHO}$ performed in standing horses, which can reduce anesthetic complications but not the complications of the procedure.

Supportive medical treatment with anti-inflammatory drugs were used to decrease inflammation on the site and broad-spectrum antibiotic therapy was used to prevent any infection on the surgical site. A secondary corneal ulcer was present and treated with topical antimicrobials and autologous serum, which has an antiproteinase activity avoiding the melting of the cornea [3]. The antimicrobial was changed once no improvement was identified in the first few days of treatment.

The treatment plan also included electro acupuncture sessions to stimulate the facial nerve.
Electro stimulation of acupuncture points can produce analgesia and neural repair, being used as a therapeutic option for different muscular and neurologic conditions including facial paralysis [7,9]. Although the mare of this report didn't tolerate the needles insertion in the facial points to perform more sessions, facial nerve paralysis improved until the time of its discharge, remaining with only a residual ptosis of the ear. The authors believe that this complementary treatment approach could be interesting to further improve the outcome in the disease, once some authors report 8 weeks to 2 years for improvement or complete resolution of neurological deficits including facial paralysis in the majority of cases [17,21].

In conclusion, despite facing one complication during $\mathrm{CHO}$, the procedure was successfully performed and well tolerated by the mare. Neurologic signs improved significantly until her discharge as well as the corneal ulcer, which was completely healed. The remaining ptosis of the ear had complete resolution within 3 months. This report reaffirms the importance of a complete diagnosis of the disease and a consistent treatment plan to improve life quality of these patients.

\section{MANUFACTURERS}

${ }^{1}$ Ouro Fino Saúde Animal Ltda. Cravinhos, SP, Brazil.

${ }^{2}$ Ceva Saúde Animal Ltda. Juatuba, MG, Brazil.

${ }^{3}$ Brainfarma Indústria Química e Farmacêutica S.A. Anápolis, GO, Brazil.

${ }^{4}$ Jofadel Indústria Farmacêutica S.A. Varinha, SP, Brazil. ${ }^{5}$ Chemitec Agro-veterinária Ltda. São Paulo, SP, Brazil.

${ }^{6}$ J.A. Saúde Animal Indústria e Comércio de Produtos Veterinários S.A. Patrocínio Paulista, SP, Brazil.

${ }^{7}$ Syntec do Brasil Ltda. Santana de Parnaíba, SP, Brazil.

${ }^{8}$ Santisa Laboratório Farmacêutico S.A. Bauru, SP, Brazil.

${ }^{9}$ Cristália Produtos Químicos Farmacêuticos Ltda. Itapira, SP, Brazil.

${ }^{10}$ Vic Pharma Indústria e Comércio Ltda. Taquaritinga, SP, Brazil.

${ }^{11}$ Vetpharma Medicamentos Veterinários Ltda. Pelotas, RS, Brazil.

${ }^{12}$ Novartis Biociências S.A. São Paulo, SP, Brazil.

Declaration of interest. The authors report no conflicts of interest. The authors alone are responsible for the content and writing of paper.

\section{REFERENCES}

1 Blythe L.L. 1997. Otitis media and interna and temporohyoid osteoarthropathy. Veterinary Clinics of North America: Equine Practice. 13(1): 21-42.

2 Bras J.J., Davis E. \& Beard W.L. 2014. Bilateral ceratohyoidectomy for the resolution of clinical signs associated with temporohyoid osteoarthropathy. Equine Veterinary Education. 26(3): 116-120.

3 Brooks D.E. 2002. Equine Ophthalmology. In: Proceedings of the 48th Annual Convention of the AAEP (Florida, U.S.A.). pp.300-313. 
4 Divers T.J., Ducharme N.G., Lahunta A., Irby N.L. \& Scrivani P.V. 2006. Temporohyoid Osteoarthopathy. Clinical Techniques in Equine Practice. 5: 17-23.

5 Espinosa P.M., Nieto J.E., Estell K.E., Kass P.H. \& Aleman M. 2017. Outcome after medical and surgical intervention in horses with temporohyoid osteoarthropathy. Equine Veterinary Journal. 49(6): 770-775.

6 Freeman D. \& Hardy J. 2012. Gutural Pouch. In: Auer J.A. \& Stick J.A. (Eds). Equine Surgery. 4th edn. St. Louis: Saunders Elsevier, pp.623-642.

7 Foirmestraux C., Tessier C. \& Touzot-Jourde G. 2014. Multimodal therapy including electroacupuncture for the treatment of facial nerve paralysis in a horse. Equine Veterinary Education. 26(1): 18-23.

8 Grenager N.S., Divers T.J., Mohmmed H.O., Johnson A.L., Albright J. \& Reuss S.M. 2010. Epidemiological features and association with crib-biting in horses with neurological disease associated with temporohyoid osteoarthropathy (1991-2008). Equine Veterinary Education. 22(9): 467-472.

9 Gülanber G.E. 2008. The Clinical Effectiveness and Application of Veterinary Acupuncture. American Journal of Traditional Chinese Veterinary Medicine. 3(1): 9-22.

10 Hilton H., Puchalski S.M. \& Aleman M. 2009. The computed tomographic appearance of equine temporohyoid osteoarthropathy. Veterinary Radiology \& Ultrasound. 50(2): 151-156.

11 Inui K.Y., Itoh M. Yanagawa M., Higuchi T., Watanabe A., Imamura Y., Urabe M. \& Sasaki N. 2017. Computed tomography and magnetic resonance imaging findings for the initial stage of equine temporohyoid osteoarthropathy in a Thoroughbred foal Tomohiro. Journal of Equine Science. 28(3): 117-121.

12 Koch C. \& Witte T. 2014. Temporohyoid osteoarthropathy in the horse. Equine Veterinary Education. 26(3): 121-125.

13 Oliver S.T. \& Hardy J. 2015. Ceratohyoidectomy for treatment of equine temporohyoid osteoarthopathy (15 cases). The Canadian Veterinary Journal. 56(4): 382-386.

14 Palus V., Bladon B., Brazil T., Cherubini G.B., Powell S.E., Greet T.R.C. \& Marr C.M. 2011. Retrospective study of neurological signs and management of seven English horses with temporohyoid osteoarthropathy. Equine Veterinary Education. 24(8): 415-422.

15 Pease A.P., Van Biervliet N.L., Dykes T.J., Divers T.J. \& Ducharme N.G. 2004. Complication of partial stylohyoidectomy for treatment of temporohyoid osteoarthropathy and an alternative surgical technique in three cases. Equine Veterinary Journal. 36(6): 546-550.

16 Racine J. O’brien T., Blandon B.M., Cruz A.M., Stoffel M.H., Haenssgen K., Rodgerson D.H., Livesey M.A. \& Koch C. 2019. Ceratohyoidectomy in standing sedated horses. Veterinary Surgery. 48(8): 1391-1398.

17 Readford P.K., Lester G.D. \& Secombe C.J. 2013. Temporohyoid osteoarthropathy in two young horses. Australian Veterinary Journal. 91(5): 209-212.

18 Rush B.R. \& Grady J.A. 2009. Vestibular Disease: Temporohyoid Osteoarthropathy. Compendium Equine: Continuing Education for Veterinarians. 4(6): 278-282.

19 Saito Y. \& Amaya T. 2019. Symptoms and management of temporohyoid osteoarthropathy and its association with crib-biting behavior in 11 Japanese Thoroughbreds. Journal of Equine Science. 30(4): 81-85.

20 Speirs V.C. 1999. Exame Clínico de Equinos. Porto Alegre: Artmed, 366p.

21 Walker A.M., Sellon D.C., Cornelisse C.J., Hines M.T., Ragle C.A., Cohen N. \& Schott II H.C. 2002. Temporohyoid Osteoarthropathy in 33 Horses (1993-2000). Journal of Veterinary Internal Medicine. 16(6): 697-703. 